\title{
Oncolytic Virotherapy for Hematological Malignancies
}

\author{
Swarna Bais, ${ }^{1}$ Eric Bartee, ${ }^{2}$ Masmudur M. Rahman, ${ }^{2}$ \\ Grant McFadden, ${ }^{2}$ and Christopher R. Cogle ${ }^{1}$ \\ ${ }^{1}$ Division of Hematology and Oncology, Department of Medicine, College of Medicine, University of Florida, ARB R4-202, \\ P.O. Box 100278, Gainesville, FL 32610-0278, USA \\ ${ }^{2}$ Department of Molecular Genetics and Microbiology, College of Medicine, University of Florida, ARB R4-295, P.O. Box 100266, \\ Gainesville, FL 32610, USA
}

Correspondence should be addressed to Grant McFadden, grantmcf@ufl.edu and Christopher R. Cogle, c@ufl.edu

Received 6 July 2011; Accepted 31 August 2011

Academic Editor: Nanhai G. Chen

Copyright (๑) 2012 Swarna Bais et al. This is an open access article distributed under the Creative Commons Attribution License, which permits unrestricted use, distribution, and reproduction in any medium, provided the original work is properly cited.

\begin{abstract}
Hematological malignancies such as leukemias, lymphomas, multiple myeloma (MM), and the myelodysplastic syndromes (MDSs) primarily affect adults and are difficult to treat. For high-risk disease, hematopoietic stem cell transplant (HCT) can be used. However, in the setting of autologous HCT, relapse due to contamination of the autograft with cancer cells remains a major challenge. Ex vivo manipulations of the autograft to purge cancer cells using chemotherapies and toxins have been attempted. Because these past strategies lack specificity for malignant cells and often impair the normal hematopoietic stem and progenitor cells, prior efforts to ex vivo purge autografts have resulted in prolonged cytopenias and graft failure. The ideal ex vivo purging agent would selectively target the contaminating cancer cells while spare normal stem and progenitor cells and would be applied quickly without toxicities to the recipient. One agent which meets these criteria is oncolytic viruses. This paper details experimental progress with reovirus, myxoma virus, measles virus, vesicular stomatitis virus, coxsackievirus, and vaccinia virus as well as requirements for translation of these results to the clinic.
\end{abstract}

\section{Hematological Malignancies}

Hematological malignancies include leukemias, lymphomas, multiple myeloma (MM), and the myelodysplastic syndromes (MDSs) that most often affect individuals older than 60 years of age. These blood cancers affect approximately $10 \%$ of Americans diagnosed with cancer each year, and an estimated 140,000 were newly diagnosed in 2010 (National Cancer Institute, Surveillance Epidemiology, and End Results). Unfortunately, despite best available therapies, an estimated 50,000 individuals died from these diseases in 2010.

The causes of hematological cancers vary depending on the specific malignancy. Exposure to environmental toxins such as benzenes, prior cytotoxic treatment such as radiotherapy or chemotherapy for an antecedent cancer, as well as infections have all been implicated as causative factors in initiating hematological malignancies. In contrast, recurrent cytogenetic abnormalities have also been observed in hematological malignancies. These abnormalities often form the basis for assigning prognosis. For example, in acute myeloid leukemia (AML), recurrent mutations that portend for a high risk of relapse after conventional treatment include those with chromosome 7 abnormalities, chromosome 5 abnormalities, complex karyotypic abnormalities, and mutations in the FLT3 gene. Genetic information can also indicate the most appropriate therapy. For instance, in patients with acute promyelocytic leukemia with the abnormal $P M L$ RARA gene fusion, treatment with all transretinoic acid (ATRA) and cytotoxic chemotherapy can cure approximately $90 \%$ of patients [1]. In patients with MDS and deletion of chromosome $5 \mathrm{q}$, treatment with lenalidomide can improve blood counts in $75 \%$ of patients [2].

Based on the utility of genetic information in determining prognosis and type of treatment in hematological malignancies, increased attention has been given to fully assessing the blood cancer genome. Recently, whole genome sequencing of an AML patient's DNA revealed several novel mutations never before associated with oncogenesis [3]. This technology also recently led to the discovery of TET2 
mutations as common gene mutations in MDS and emphasized the importance of epigenetic dysregulation in this disease $[4,5]$. Because of the abnormal DNA methylation that occurs after TET2 mutations, finding this mutation in an MDS patient's genome may indicate treatment with a hypomethylating agent such as azacitidine or decitabine [6]. Recently, whole genome sequencing was reported useful in determining the best treatment for a patient with AML [7]. Thus, genome analysis has the strong potential for personalized medicine in hematological malignancies.

In some hematological malignancies, such as MDS, abnormalities in bone marrow stromal cells are believed to affect hematopoietic stem and progenitor cells, leading to neoplastic transformation [8]. Evidence that the bone marrow microenvironment is an important factor in the oncogenesis of hematological malignancies has spurred great interest in regulating microenvironmental interactions as a means for improved therapies. We have targeted blood vessels in the leukemia niche with the novel vascular disrupting combretastatin, OXi4503, and have successfully regressed disease [9]. This work has been translated into a phase I clinical study (http://www.ClinicalTrials.gov Identifier NCT01085656).

Cancer stem cells have been identified for some hematological malignancies [10]. In the specific case of acute myeloid leukemia (AML), a small subpopulation of cancer stem cells have been identified in the CD34+CD38-CD123+ fraction [11, 12]. In MM, myeloma stem cells have been found in the CD138- B cell fraction, which replicate and differentiate into CD138+ malignant plasma cells [13]. In chronic myeloid leukemia (CML), hematopoietic progenitor cells are believed to be the cancer-initiating cells which are endowed with cancer stem cell properties after acquiring the abnormal $B C R / A B L$ gene fusion [14]

\section{Treatment of Hematological Malignancies}

The cornerstone of conventional therapy for hematological malignancies includes agents that block cell division such as antimetabolites (e.g., cytarabine), DNA alkylating agents (e.g., cyclophosphamide), and anthracyclines (e.g., daunorubicin). Treatment with these agents induces initial remission in a high percentage of patients; however, relapsed disease remains a major challenge in treating patients with hematological malignancies.

For example, in cases of AML, remission rates with standard induction chemotherapy such as seven days of continuously infused cytarabine and three days of anthracycline bring about initial complete remissions in approximately 30$70 \%$ of patients. However, in older individuals, who are more commonly diagnosed with AML, long-term prognosis can be grim with only $10-20 \%$ of patients surviving without disease [15]. For patients with high risk AML, allogeneic HCT is used and can be curative in approximately $40 \%$ of patients. With this procedure, the donor immune system recognizes any residual leukemia in the recipient as foreign because of minor human leukocyte antigen mismatches and/or unique AML antigens, resulting in elimination and persistent surveillance for the malignant cells. By similar mechanisms, the donor immune system can recognize the recipient's normal organs (skin, gastrointestinal tract, liver, lungs, joints) as foreign and elicit graft versus host disease (GVHD). Although potentially curative, most AML patients due to their age-related comorbidities are not fit for the high risks associated with allogeneic HCT (e.g., GVHD, lifethreatening infections, organ toxicity) and/or do not have a suitable allogeneic stem cell donor. Experimental therapies for AML have recently included specific mutation-targeting agents such as FLT3 inhibitors for patients with internal tandem duplications in the FLT3 gene of the AML clone. However, results from these clinical trials have been disappointing.

For patients with MM, treatment decisions are often based on risk for refractory and relapsed disease. Certain chromosome abnormalities, such as deletion of chromosome 13 , portend for poor prognosis. In addition, gene expression profiling can be used to risk-stratify MM disease [16]. For patients with standard risk MM, initial treatment is dependent on the patient's eligibility for high-dose chemotherapy followed by autologous HCT, which can prolong diseasefree and overall survival but carries treatment-related risks of organ toxicity, need for transfusions, and life-threatening infection. Patients eligible for autologous HCT are treated with nonalkylating agent induction therapies such as thalidomide and dexamethasone or lenalidomide and dexamethasone $[17,18]$. After this initial therapy, patients have the option of early versus delayed autologous HCT. If early HCT is used, then a second HCT can be performed in patients who do not achieve a very good partial remission or better [19]. If the patient elects for delayed high-dose chemotherapy followed by autologous HCT, then transplant is not performed until initial induction therapy brings about a plateau in response or progressed disease develops. Even using these treatments, however, autologous HCT rarely brings about cures for MM, as the disease nearly always relapses.

\section{Autograft Contamination and Disease Relapse after Transplant}

Despite the significant increase in use of autologous HCT for hematologic malignancies, disease relapse is a primary cause of death after transplant. Graft contamination is thought to be the chief reason for posttransplant relapse. This premise is supported by multiple lines of evidence. First, transplant of HSPC from syngeneic (identical twin) donors leads to lower incidences of disease relapse in patients with multiple myeloma, low-grade non-Hodgkin's lymphoma, AML, and ALL [20-22]. Second, numerous reports show that transplanted autografts contain minimal residual disease (MRD) in a variety of patients with cancer [23-39]. The level of MRD, detected by flow cytometry, immunohistochemistry, and molecular methods, directly correlated with risk of disease relapse and death. Whereas these lines of evidence show a strong correlation, direct proof of contaminated autografts through tracing studies are most compelling. Thus, the third line of evidence comes from gene 
marking studies [40-42]. In these clinical studies, autologous HSPCs were genetically tagged and then transplanted. Relapsed disease was evaluated for the tag. In a variety of leukemias and cancers, the posttransplant relapsed disease contained the pretransplant tag. Together, these lines of evidence support the premise that contaminating cells within the autologous transplant graft can be the origin of relapsed disease after transplant.

\section{Purging Strategies}

Considering the high rates of refractory and relapsed in patients with hematological malignancies and evidence of contaminating cancer cells in autologous HCT grafts, it is possible that graft purging of contaminating cells may improve posttransplant disease-free and overall survival. Ideally, a safe and effective purging strategy should specifically target the contaminating malignant clone and spare normal HSPC needed for reconstitution of immunity, erythropoieis, and platelets.

Several purging strategies have been attempted to selectively target malignant cells from autologous HCT grafts. One strategy is to treat the autologous graft after collection but prior to transplant back into the patient. A number of these ex vivo purging techniques have been tested such as:

(i) chemotherapy with antiproliferative drugs such mafosfamide and 4-hydroperoxycyclophosphamide (4$\mathrm{HC}$, active metabolite of cyclophosphamide) [43, 44];

(ii) CD34+ stem/progenitor cell enrichment using immunomagnetic selection [45];

(iii) immunotoxins or hybrid cytotoxic proteins designed to selectively kill cancer cells such as heregulin (HRG) Pseudomonas exotoxin (PE) 40 [46];

(iv) immunomagnetic removal of tumor cells when the tumor cells express a unique antigen [47];

(v) monoclonal antibodies such as alemtuzumab (antiCD52) and rituximab (anti-CD20) [48];

(vi) photodynamic purging by rhodamine [49].

Unfortunately most of these ex vivo purging techniques also impaired normal hematopoietic stem and progenitor (HSPC) function and therefore have not translated to routine clinical practice for autologous HCT [50]. Purging strategies utilizing cytotoxic chemotherapy can be nonselective to cancer cells, and HSPC can be susceptible to the cytotoxic drugs $[43,44]$. Immunomagnetic selection based on one cell surface marker (i.e., CD34) can enrich for normal HSPC; however, this selection process is never $100 \%$, and the positive fraction may contain contaminating cancer cells $[45,47]$. Moreover, discriminating normal from malignant HSPC can be difficult when using just cell surface markers because the two populations are sometimes indistinguishable by immunophenotyping. Given similarities in immunophenotype, immunotoxins may target both malignant and normal HSPC, leading to impaired normal hematopoiesis and posttransplant hematopoietic reconstitution $[46,48]$.
Ideally, ex vivo purging is selective for the cancer cells yet spares normal HSPC. Moreover, the ideal purging technique should be applied quickly (within minutes-to-hours) so that the transplant process is not delayed and any modifications to standard transplantation protocols are minimized. Cell viability is a time-dependent variable, and the quicker the manipulation the higher cell viability for transplant. In the postthaw setting, cell viability can diminish within hours, thus purging techniques applied to thawed products should be especially time sensitive in order to provide patients with the highest cell viability for transplant.

\section{Oncolytic Virotherapy for Hematological Malignancies}

Oncolytic viruses may meet criteria as ideal purging agents for hematological malignancies. Specifically, certain oncolytic viruses selectively target malignant hematopoietic cells such as multiple myeloma and leukemia cells while sparing normal HSPCs [51]. This capacity to purge autologous HCT grafts makes oncolytic viruses particular attractive for potential use in the clinical transplant setting (Figure 1). A few oncolytic viruses have already been translated into the clinic (Table 1).

One potential purging agent is coxsackievirus A21 (CVA21) based on its ability to selectively target hematological malignant cells [52]. CVA21, a common enterovirus, exhibited a potent cytostatic and cytocidal effect against three MM cell lines with reduced cytotoxicity against normal human peripheral blood mononuclear cells (PBMCs) [53]. CVA21 specificity is believed to be related to expression of intercellular adhesion molecule-1 (ICAM-1) and decay accelerating factor (DAF) on the surface of target cells. While the immunocompromised status of MM patients receiving chemotherapy poses a concern for the use of virotherapy, it may be in these patients that CVA21 virotherapy will have the most successful outcome due to the lack of antiviral immunity. Disseminated CVA21 infection can be controlled by antiviral compounds, such as pleconaril [54] or immunoglobulin [55]. CVA21 has already been administered to end-stage melanoma patients without adverse effects [56], and further human trials are currently underway to evaluate safety.

Another potential oncolytic virus for the treatment of hematological malignancies is reovirus [57]. Reovirus is a double-stranded RNA virus that is replication competent and preferentially infects cells with hyperactivated signaling, for example, in the Ras pathway. When reovirus was used to ex vivo purge $\mathrm{MM}$ cells from admixtures of apheresis products, purging was incomplete: only 50\% of the MM cells were effectively purged. Also, reovirus was unable to purge follicular lymphoma and Burkitt's lymphoma cells [58]. A major advantage with reovirus is that it does not affect normal HSPCs. Therefore, reovirus may have potential in certain hematological malignancies, but it remains to be defined how clinically effective the virus is at eliminating each type of cancer. 


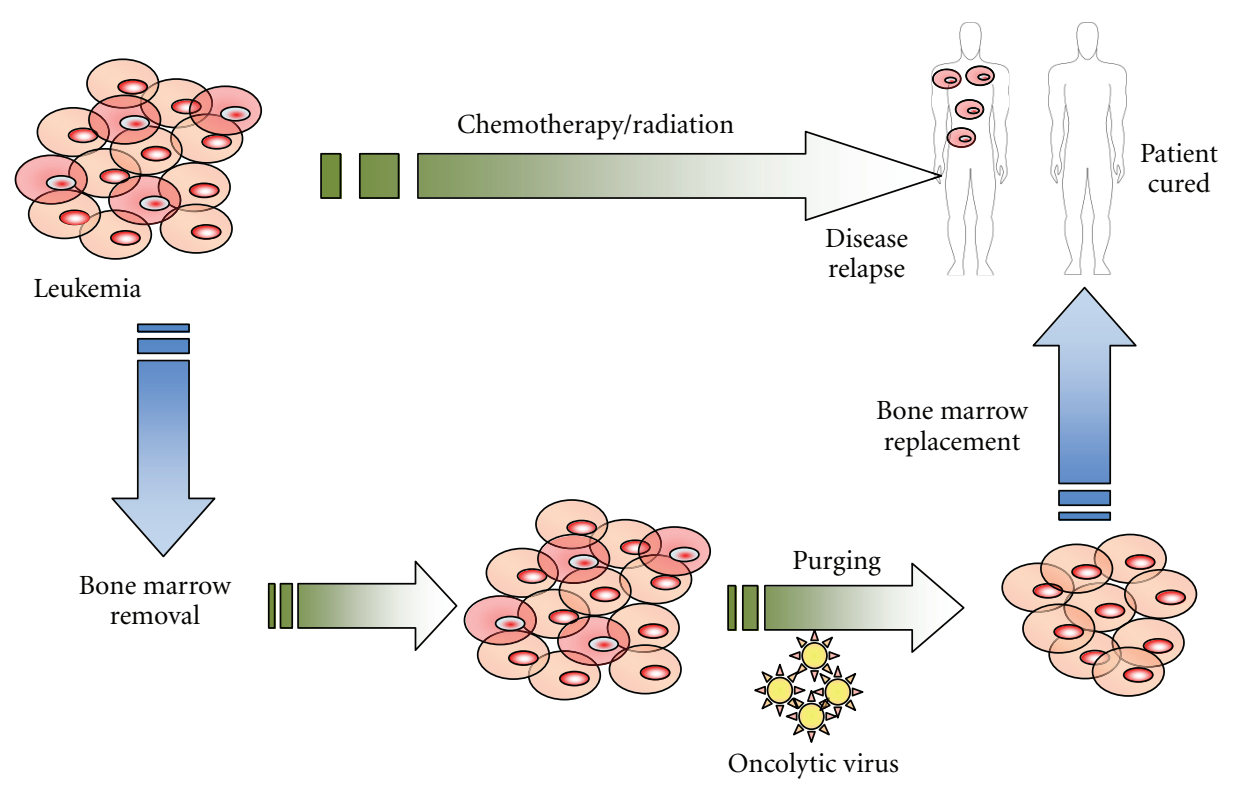

Cancer cells

Normal cells

FIGURE 1: Proposed treatment schema of oncolytic virotherapy for patients with hematological malignancies undergoing high-dose chemotherapy and autologous HCT.

Vesicular stomatitis virus (VSV) is another virus with oncolytic potential [59]. This negative strand RNA virus lacks toxicity for HSPCs in culture and has oncolytic activity against AML cell lines. Moreover, VSV can purge MM from mobilized PBSC CD34+ cells [60].

The Edmonston-B vaccine strain of measles virus (MVEdm) also has reported oncolytic activity against MM. Using six clinical MM samples and a transplant model into immunodeficient mice, this measles virus successfully purged myeloma cells [61]. The intrinsic tumor selective cytotoxicity is an attractive feature of this agent. They also noted that administration of MV-Edm into MV-susceptible transgenic mice expressing the human CD46 receptor resulted in infection of macrophages in spleen, lymph nodes, and peritoneal cavity [62]. To enhance virus specificity, they generated an anti-CD38 scFv and demonstrated that display of scFv redirected virus binding and entry into $\mathrm{CD} 38$ receptor positive cells that were devoid of natural measles receptors [63]. The MV-Edm virus is currently in a phase I clinical study for recurrent or refractory MM where it is administered systemically via intravenous route along with cyclophosphamide chemotherapy (http://www.ClinicalTrials.gov ID NCT00450814) [64]. In this trial, the investigators are using the MV-NIS Edmonston lineage which was genetically engineered to express the human sodium iodide symporter (NIS). Insertion of the NIS protein into MV enables pharmacokinetic monitoring of the virus by means of radioactive iodine $\left({ }^{123} \mathrm{I}\right)$ administration. Cells infected with MV-NIS will show increased uptake of the radioactive iodine, and this uptake can be serially tracked in real time. The patient's
TABLE 1: Oncolytic viruses for the treatment of hematological malignancies.

\begin{tabular}{lccc}
\hline Virus & Disease targets & Clinical studies & References \\
\hline Reovirus & MM, NHL, CLL & In development & {$[57]$} \\
Myxoma virus & AML, MM & In development & {$[51]$} \\
Measles virus & MM & Ongoing & {$[64]$} \\
Vaccinia virus & MM & Case report & {$[76]$} \\
\hline
\end{tabular}

MM: multiple myeloma; NHL: non-Hodgkin's lymphoma; CLL: chronic lymphocytic leukemia; AML: acute myeloid leukemia.

normal thyroid function is protected by coadministration of a normal thyroid hormone, triiodothyronine (T3).

Live attenuated measles virus (MV) has potent oncolytic activity against MM tumor xenografts. The virus is tumor selective and preferentially targets cells that express high levels of CD46 receptors [65]. A vaccine strain of MV causes regression of large established human lymphoma xenografts in immunodeficient mice. MV is a negative-strand RNA virus, and, interestingly, the presence of anti-MV antibodies does not compromise the oncolytic effect of MV [66].

Adachi et al. reported a midkine promoter based conditionally replicative adenovirus (Ad) for the treatment of pediatric solid tumors and bone marrow tumor purging. A conditionally replicative Ad in which the expression of E1 is controlled by the MK promoter achieved high levels of viral replication in neuroblastoma or Ewing's sarcoma cells and induced tumor cell killing. No damage to CD34+ cells was 
seen, even after three hours of infection at 1000 MOI [67]. Adenovirus serotype 5 (Ad5) and other low-seroprevalence adenoviruses may have utility as oncolytic agents against $\mathrm{MM}$ and other hematological malignancies [68].

Tumor-specific double-deleted Vaccinia virus has also been tested in multiple myeloma [69]. Esfandyari et al. were the first to document permissiveness of lymphoma cells to oncolytic herpes viruses and introduced ELK as a suitable factor for predicting tumor susceptibility to novel anticancer agents [70].

Oncolytic rat parvovirus, $\mathrm{H}-1 \mathrm{PV}$, may be a potential candidate for the treatment of some non-Hodgkin's B-cell lymphomas, including those resistant to apoptosis induction by rituximab. $\mathrm{H}-1 \mathrm{PV}$ efficiently killed through necrosis while sparing normal B lymphocytes [71].

Recently, we showed that myxoma virus (MYXV) has the capacity to selectively target primary human leukemia cells while spare normal HSPCs [51]. Poxviruses such as MYXV can bind and initiate entry into most mammalian cells but then discriminates permissive versus nonpermissive cells by virtue of the cell signaling circuitry of the infected cell. We have shown that upregulated AKT signaling, either as constitutive phosphorylation or induced by virus infection [72], regulates MYXV permissiveness in a wide variety of human solid tumor cell lines [73]. Considering the complexity and heterogeneity of cancer cells, this pathway is likely not the only mechanism for cancer cell specificity and there may be other mechanisms to explain the virus' discrimination between leukemia cells and normal HSPC. For example, when normal macrophages are infected with MYXV, the cells rapidly coinduce two antiviral cytokines (tumor necrosis factor and type I interferon) by a RIG-I-dependent signaling mechanism, which then aborts MYXV infection in normal somatic cells in a paracrine-like manner [74]. Thus, it could be that normal HSPCs are competent for this synergy, whereas malignant HSPCs, such as AML cells, are defective in some aspect of the tumor necrosis factor/interferon pathway. The mechanism for selective killing of cancer is still being studied, but two important factors include (1) most human cancer cells lack type I IFN and TNF synergy responses [75] and (2) most cancer cells have excessive levels of activated Akt, which facilitates MYXV replication [73].

\section{Clinical Translation of Oncolytic Viruses as Purging Agents for HCT}

For successful clinical translation, there are some unique requirements for oncolytic virotherapy in the setting of purging cancer cells prior to HCT. First, the OV must spare normal HSPCs. Second, the purging strategy should be simple and quick, especially when using cryopreserved stem cell products. After thawing autologous cryopreserved HSPC, cell viability decreases quickly (within minutes to an hour); thus any postthaw intervention must be quickly performed to ensure transplant of an adequate number of viable HSPC. Finally, the oncolytic virus must show limited to no infection of recipient somatic cells or tissues considering that all transplant recipients are highly immunocompromised after high-dose chemotherapy and autologous HCT.

In addition to showing preclinical safety and efficacy, the translation of oncolytic virotherapy for hematological malignancies will also require the ability to massively scale up manufacture of clinical grade virus under good manufacturing process (GMP) conditions. This process will necessitate facilities with expertise in virus production.

Currently, there are no clinical studies of oncolytic viruses as purging agents prior to autologous HCT. However, if a virus system can be optimized to meet minimum clinical criteria, then oncolytic virotherapy would have major impact in how we treat patients with blood cancers. Already, promising experimental progress indicates that early phase clinical studies of oncolytic viruses as purging agents for HCT are imminently approaching.

\section{Acknowledgments}

This work is supported by grants from the Bankhead Coley Foundation (1BT02) and NCI (CA138541-01).

\section{References}

[1] B. U. Mueller, T. Pabst, J. Fos et al., "ATRA resolves the differentiation block in $\mathrm{t}(15 ; 17)$ acute myeloid leukemia by restoring PU.1 expression," Blood, vol. 107, no. 8, pp. 3330-3338, 2006.

[2] R. S. Komrokji and A. F. List, "Lenalidomide for treatment of myelodysplastic syndromes: current status and future directions," Hematology/Oncology Clinics of North America, vol. 24, no. 2, pp. 377-388, 2010.

[3] D. C. Link, L. G. Schuettpelz, D. Shen et al., "Identification of a novel TP53 cancer susceptibility mutation through wholegenome sequencing of a patient with therapy-related AML," Journal of the American Medical Association, vol. 305, no. 15, pp. 1568-1576, 2011.

[4] M. Ko, Y. Huang, A. M. Jankowska et al., "Impaired hydroxylation of 5-methylcytosine in myeloid cancers with mutant TET2," Nature, vol. 468, no. 7325, pp. 839-843, 2010.

[5] R. Itzykson, O. Kosmider, T. Cluzeau et al., "Impact of TET2 mutations on response rate to azacitidine in myelodysplastic syndromes and low blast count acute myeloid leukemias," Leukemia, vol. 25, no. 7, pp. 1147-1152, 2011.

[6] R. Itzykson, S. Thépot, V. Eclache et al., "Prognostic significance of monosomal karyotype in higher risk myelodysplastic syndrome treated with azacitidine," Leukemia, vol. 25, no. 7, pp. 1207-1209, 2011.

[7] J. S. Welch, P. Westervelt, L. Ding et al., "Use of whole-genome sequencing to diagnose a cryptic fusion oncogene," Journal of the American Medical Association, vol. 305, no. 15, pp. 15771584, 2011.

[8] M. H. G. P. Raaijmakers, S. Mukherjee, S. Guo et al., "Bone progenitor dysfunction induces myelodysplasia and secondary leukaemia," Nature, vol. 464, no. 7290, pp. 852-857, 2010.

[9] G. J. Madlambayan, A. M. Meacham, K. Hosaka et al., "Leukemia regression by vascular disruption and antiangiogenic therapy," Blood, vol. 116, no. 9, pp. 1539-1547, 2010.

[10] R. C. Sanders Jr., W. B. Slayton, C. R. Cogle, R. C. Fisher, and E. W. Scott, "Stem cell research," Paediatric Respiratory Reviews, vol. 7, no. 2, pp. 135-140, 2006. 
[11] J. L. McKenzie, K. Takenaka, O. I. Gan, M. Doedens, and J. E. Dick, "Low rhodamine 123 retention identifies long-term human hematopoietic stem cells within the Lin-CD34+CD38population," Blood, vol. 109, no. 2, pp. 543-545, 2007.

[12] F. Goodrum, C. T. Jordan, S. S. Terhune, K. High, and T. Shenk, "Differential outcomes of human cytomegalovirus infection in primitive hematopoietic cell subpopulations," Blood, vol. 104, no. 3, pp. 687-695, 2004.

[13] W. Matsui, C. A. Huff, Q. Wang et al., "Characterization of clonogenic multiple myeloma cells," Blood, vol. 103, no. 6, pp. 2332-2336, 2004.

[14] D. Perrotti, C. Jamieson, J. Goldman, and T. Skorski, "Chronic myeloid leukemia: mechanisms of blastic transformation," Journal of Clinical Investigation, vol. 120, no. 7, pp. 2254-2264, 2010.

[15] American Cancer Society, Cancer Facts \& Figures, The Society, Atlanta, Ga, USA, 2011.

[16] A. K. Stewart and R. Fonseca, "Prognostic and therapeutic significance of myeloma genetics and gene expression profiling," Journal of Clinical Oncology, vol. 23, no. 26, pp. 6339-6344, 2005.

[17] S. V. Rajkumar, E. Blood, D. Vesole, R. Fonseca, and P. R. Greipp, "Phase III clinical trial of thalidomide plus dexamethasone compared with dexamethasone alone in newly diagnosed multiple myeloma: a clinical trial coordinated by the eastern cooperative oncology group," Journal of Clinical Oncology, vol. 24, no. 3, pp. 431-436, 2006.

[18] S. V. Rajkumar, S. Jacobus, N. S. Callander et al., "Lenalidomide plus high-dose dexamethasone versus lenalidomide plus low-dose dexamethasone as initial therapy for newly diagnosed multiple myeloma: an open-label randomised controlled trial," The Lancet Oncology, vol. 11, no. 1, pp. 2937, 2010.

[19] B. Barlogie, M. Attal, J. Crowley et al., "Long-term followup of autotransplantation trials for multiple myeloma: update of protocols conducted by the intergroupe francophone du myelome, southwest oncology group, and university of arkansas for medical sciences," Journal of Clinical Oncology, vol. 28, no. 7, pp. 1209-1214, 2010.

[20] G. Gahrton, H. Svensson, B. Björkstrand et al., "Syngeneic transplantation in multiple myeloma-a case-matched comparison with autologous and allogeneic transplantation," Bone Marrow Transplantation, vol. 24, no. 7, pp. 741-745, 1999.

[21] V. Lj Lazarevic, H. Högglund, M. Remberger et al., "Longterm survival following allogeneic or syngeneic stem cell transplant for follicular lymphoma in Sweden," Leukemia and Lymphoma, vol. 52, no. 1, pp. 69-71, 2011.

[22] L. Fouillard, M. Labopin, A. Gratwohl et al., "Results of syngeneic hematopoietic stem cell transplantation for acute leukemia: risk factors for outcomes of adults transplanted in first complete remission," Haematologica, vol. 93, no. 6, pp. 834-841, 2008.

[23] W. Vogel, H. G. Kopp, L. Kanz, and H. Einsele, "Myeloma cell contamination of peripheral blood stem-cell grafts can predict the outcome in multiple myeloma patients after high-dose chemotherapy and autologous stem-cell transplantation," Journal of Cancer Research and Clinical Oncology, vol. 131, no. 4, pp. 214-218, 2005.

[24] S. Galimberti, F. Morabito, F. Guerrini et al., "Peripheral blood stem cell contamination evaluated by a highly sensitive molecular method fails to predict outcome of autotransplanted multiple myeloma patients," British Journal of Haematology, vol. 120, no. 3, pp. 405-412, 2003.
[25] A. M. Barbui, M. Galli, G. Dotti et al., "Negative selection of peripheral blood stem cells to support a tandem autologous transplantation programme in multiple myeloma," British Journal of Haematology, vol. 116, no. 1, pp. 202-210, 2002.

[26] J. W. Friedberg, H. Kim, S. Li et al., "Ex vivo B cell depletion using the Eligix B cell SC system and autologous peripheral blood stem cell transplantation in patients with follicular nonHodgkin's lymphoma," Bone Marrow Transplantation, vol. 32, no. 7, pp. 681-686, 2003.

[27] L. De Rosa, M. Lalle, A. Pandolfi, C. Ruscio, and R. Amodeo, "Autologous bone marrow transplantation with negative immunomagnetic purging for aggressive B-cell non-Hodgkin's lymphoma in first complete remission," Annals of Hematology, vol. 81, no. 10, pp. 575-581, 2002.

[28] S. Galimberti, R. Marasca, F. Caracciolo et al., "The role of molecular monitoring in autotransplantation for non-Hodgkin's lymphoma," Bone Marrow Transplantation, vol. 29, no. 7, pp. 581-587, 2002.

[29] J. C. McCann, R. Kanteti, B. Shilepsky, K. B. Miller, M. Sweet, and D. P. Schenkein, "High degree of occult tumor contamination in bone marrow and peripheral blood stem cells of patients undergoing autologous transplantation for non-Hodgkin's lymphoma," Biology of Blood and Marrow Transplantation, vol. 2, no. 1, pp. 37-43, 1996.

[30] N. Feller, M. A. Van Der Pol, T. Waaijman et al., "Immunologic purging of autologous peripheral blood stem cell products based on CD34 and CD133 expression can be effectively and safely applied in half of the acute myeloid leukemia patients," Clinical Cancer Research, vol. 11, no. 13, pp. 4793-4801, 2005.

[31] T. Miyamoto, K. Nagafuji, M. Harada et al., "Quantitative analysis of AML1/ETO transcripts in peripheral blood stem cell harvests from patients with $\mathrm{t}(8 ; 21)$ acute myelogenous leukaemia," British Journal of Haematology, vol. 91, no. 1, pp. 132-138, 1995.

[32] E. S. Sandler, A. Homans, L. Mandell et al., "Hematopoietic stem cell transplantation after first marrow relapse of nonT, non-B acute lymphoblastic leukemia: a Pediatric Oncology Group pilot feasibility study," Journal of Pediatric Hematology/Oncology, vol. 28, no. 4, pp. 210-215, 2006.

[33] J. Atta, F. Fauth, M. Keyser et al., "Purging in BCR-ABLpositive acute lymphoblastic leukemia using immunomagnetic beads: comparison of residual leukemia and purging efficiency in bone marrow vs peripheral blood stem cells by semiquantitative polymerase chain reaction," Bone Marrow Transplantation, vol. 25, no. 1, pp. 97-104, 2000.

[34] W. Leung, A. R. Chen, R. C. Klann et al., "Frequent detection of tumor cells in hematopoietic grafts in neuroblastoma and Ewing's sarcoma," Bone Marrow Transplantation, vol. 22, no. 10, pp. 971-979, 1998.

[35] S. A. Burchill et al., "Minimal residual disease at the time of peripheral blood stem cell harvest in patients with advanced neuroblastoma," Medical and Pediatric Oncology, vol. 36, no. 1, pp. 213-219, 2001.

[36] R. Schulze, M. Schulze, A. Wischnik et al., "Tumor cell contamination of peripheral blood stem cell transplants and bone marrow in high-risk breast cancer patients," Bone Marrow Transplantation, vol. 19, no. 12, pp. 1223-1228, 1997.

[37] A. M. Vannucchi, A. Bosi, S. Glinz et al., "Evaluation of breast tumour cell contamination in the bone marrow and leukapheresis collections by RT-PCR for cytokeratin-19 mRNA," British Journal of Haematology, vol. 103, no. 3, pp. 610-617, 1998.

[38] C. Bokemeyer, A. J.M. Gillis, K. Pompe et al., "Clinical impact of germ cell tumor cells in apheresis products of patients 
receiving high-dose chemotherapy," Journal of Clinical Oncology, vol. 19, no. 12, pp. 3029-3036, 2001.

[39] T. Kasahara, N. Hara, V. Bilim et al., "Sequential detection of alphafetoprotein-bearing cells in blood stem cell fraction of germ cell tumour patients," British Journal of Cancer, vol. 85, no. 8, pp. 1119-1123, 2001.

[40] M. K. Brenner, D. R. Rill, R. C. Moen et al., "Gene-marking to trace origin of relapse after autologous bone-marrow transplantation," The Lancet, vol. 341, no. 8837, pp. 85-86, 1993.

[41] A. B. Deisseroth, Z. Zu, D. Claxton et al., "Genetic marking shows that $\mathrm{Ph}+$ cells present in autologous transplants of chronic myelogenous leukemia (CML) contribute to relapse after autologous bone marrow in CML," Blood, vol. 83, no. 10, pp. 3068-3076, 1994.

[42] D. R. Rill, V. M. Santana, W. M. Roberts et al., "Direct demonstration that autologous bone marrow transplantation for solid tumors can return a multiplicity of tumorigenic cells," Blood, vol. 84, no. 2, pp. 380-383, 1994.

[43] M. R. Motta, S. Mangianti, S. Rizzi et al., "Pharmacological purging of minimal residual disease from peripheral blood stem cell collections of acute myeloblastic leukemia patients: preclinical studies," Experimental Hematology, vol. 25, no. 12, pp. 1261-1269, 1997.

[44] E. J. Shpall, R. B. Jones, R. C. Bast et al., "4-Hydroperoxycyclophosphamide purging of breast cancer from the mononuclear cell fraction of bone marrow in patients receiving high-dose chemotherapy and autologous marrow support: a phase I trial," Journal of Clinical Oncology, vol. 9, no. 1, pp. 8593, 1991.

[45] R. Abonour, K. M. Scott, L. A. Kunkel et al., "Autologous transplantation of mobilized peripheral blood CD34+ cells selected by immunomagnetic procedures in patients with multiple myeloma," Bone Marrow Transplantation, vol. 22, no. 10, pp. 957-963, 1998.

[46] A. Spyridonidis, M. Schmidt, W. Bernhardt et al., "Purging of mammary carcinoma cells during ex vivo culture of CD34+ hematopoietic progenitor cells with recombinant immunotoxins," Blood, vol. 91, no. 5, pp. 1820-1827, 1998.

[47] A. Gee, T. Moss, V. Mansour et al., "Large-scale immunomagnetic separation system for the removal of tumor cells from bone marrow," Progress in Clinical and Biological Research, vol. 377, pp. 181-187, 1992.

[48] B. D. Cheson, "Monoclonal antibody therapy for B-cell malignancies," Seminars in Oncology, vol. 33, no. 2, supplement 5, pp. 2-14, 2006.

[49] T. F. Goggins and N. Chao, "Depletion of host reactive T cells by photodynamic cell purging and prevention of graft versus host disease," Leukemia and Lymphoma, vol. 44, no. 11, pp. 1871-1879, 2003.

[50] S. Y. Wang, K. L. Makl, L. Y. Chen, C. H. Tzeng, C. P. Hu, and C. Chang, "Elimination of malignant tumor cells from human bone marrow using monoclonal antibodies and immunomagnetic beads," Anticancer Research, vol. 13, no. 6 A, pp. 2281-2285, 1993.

[51] M. Kim, G. J. Madlambayan, M. M. Rahman et al., "Myxoma virus targets primary human leukemic stem and progenitor cells while sparing normal hematopoietic stem and progenitor cells," Leukemia, vol. 23, no. 12, pp. 2313-2317, 2009.

[52] G. G. Au, L. F. Lincz, A. Enno, and D. R. Shafren, "Oncolytic Coxsackievirus A21 as a novel therapy for multiple myeloma," British Journal of Haematology, vol. 137, no. 2, pp. 133-141, 2007.

[53] D. R. Shafren, D. J. Dorahy, S. J. Greive, G. F. Burns, and R. D. Barry, "Mouse cells expressing human intercellular adhesion molecule- 1 are susceptible to infection by coxsackievirus A21," Journal of Virology, vol. 71, no. 1, pp. 785-789, 1997.

[54] J. M. Rogers, G. D. Diana, and M. A. McKinlay, "Pleconaril: a broad spectrum antipicornaviral agent," Advances in Experimental Medicine and Biology, vol. 458, pp. 69-76, 1999.

[55] H. A. Rotbart, "Antiviral therapy for enteroviral infections," Pediatric Infectious Disease Journal, vol. 18, no. 7, pp. 632-633, 1999.

[56] K. A. Parato, D. Senger, P. A. J. Forsyth, and J. C. Bell, "Recent progress in the battle between oncolytic viruses and tumours," Nature Reviews Cancer, vol. 5, no. 12, pp. 965-976, 2005.

[57] C. M. Thirukkumaran, J. M. Luider, D. A. Stewart et al., "Reovirus oncolysis as a novel purging strategy for autologous stem cell transplantation," Blood, vol. 102, no. 1, pp. 377-387, 2003.

[58] T. Alain, K. Hirasawa, K. J. Pon et al., "Reovirus therapy of lymphoid malignancies," Blood, vol. 100, no. 12, pp. 41464153, 2002.

[59] D. F. Stojdl, B. Lichty, S. Knowles et al., "Exploiting tumorspecific defects in the interferon pathway with a previously unknown oncolytic virus," Nature Medicine, vol. 6, no. 7, pp. 821-825, 2000.

[60] S. Deola, S. Scaramuzza, R. S. Birolo et al., "Molecular purging of multiple myeloma cells by ex-vivo culture and retroviral transduction of mobilized-blood CD34+ cells," Journal of Translational Medicine, vol. 5, article no. 35, 2007.

[61] K. W. Peng, G. J. Ahmann, L. Pham, P. R. Greipp, R. Cattaneo, and S. J. Russell, "Systemic therapy of myeloma xenografts by an attenuated measles virus," Blood, vol. 98, no. 7, pp. 20022007, 2001.

[62] B. Roscic-Mrkic, R. A. Schwendener, B. Odermatt et al., "Roles of macrophages in measles virus infection of genetically modified mice," Journal of Virology, vol. 75, no. 7, pp. 33433351, 2001.

[63] K. W. Peng, K. A. Donovan, U. Schneider, R. Cattaneo, J. A. Lust, and S. J. Russell, "Oncolytic measles viruses displaying a single-chain antibody against CD38, a myeloma cell marker," Blood, vol. 101, no. 7, pp. 2557-2562, 2003.

[64] R. M. Myers, S. M. Greiner, M. E. Harvey et al., "Preclinical pharmacology and toxicology of intravenous MV-NIS, an oncolytic measles virus administered with or without cyclophosphamide," Clinical Pharmacology and Therapeutics, vol. 82, no. 6, pp. 700-710, 2007.

[65] H. T. Ong, M. M. Timm, P. R. Greipp et al., "Oncolytic measles virus targets high CD46 expression on multiple myeloma cells," Experimental Hematology, vol. 34, no. 6, pp. 713-720, 2006.

[66] D. Grote, S. J. Russell, T. I. Cornu et al., "Live attenuated measles virus induces regression of human lymphoma xenografts in immunodeficient mice," Blood, vol. 97, no. 12, pp. 3746-3754, 2001.

[67] Y. Adachi, P. N. Reynolds, M. Yamamoto et al., "A midkine promoter-based conditionally replicative adenovirus for treatment of pediatric solid tumors and bone marrow tumor purging," Cancer Research, vol. 61, no. 21, pp. 7882-7888, 2001.

[68] J. S. Senac, K. Doronin, S. J. Russell, D. F. Jelinek, P. R. Greipp, and M. A. Barry, "Infection and killing of multiple myeloma by adenoviruses," Human Gene Therapy, vol. 21, no. 2, pp. 179-190, 2010.

[69] H. Deng, N. Tang, A. E. Stief et al., "Oncolytic virotherapy for multiple myeloma using a tumour-specific double-deleted vaccinia virus," Leukemia, vol. 22, no. 12, pp. 2261-2264, 2008. 
[70] T. Esfandyari, A. Tefferi, A. Szmidt et al., "Transcription factors down-stream of Ras as molecular indicators for targeting malignancies with oncolytic herpes virus," Molecular Oncology, vol. 3, no. 5-6, pp. 464-468, 2009.

[71] A. L. Angelova, M. Aprahamian, G. Balboni et al., "Oncolytic rat parvovirus $\mathrm{H}-1 \mathrm{PV}$, a candidate for the treatment of human lymphoma: in vitro and in vivo studies," Molecular Therapy, vol. 17, no. 7, pp. 1164-1172, 2009.

[72] G. McFadden, "Poxvirus tropism," Nature Reviews Microbiology, vol. 3, no. 3, pp. 201-213, 2005.

[73] G. Wang, J. W. Barrett, M. Stanford et al., "Infection of human cancer cells with myxoma virus requires Akt activation via interaction with a viral ankyrin-repeat host range factor," Proceedings of the National Academy of Sciences of the United States of America, vol. 103, no. 12, pp. 4640-4645, 2006.

[74] F. Wang, X. Gao, J. W. Barrett et al., "RIG-I mediates the co-induction of tumor necrosis factor and type I interferon elicited by myxoma virus in primary human macrophages," PLoS Pathogens, vol. 4, no. 7, Article ID e1000099, 2008.

[75] E. Bartee and G. McFadden, "Human cancer cells have specifically lost the ability to induce the synergistic state caused by tumor necrosis factor plus interferon- $\beta$," Cytokine, vol. 47, no. 3, pp. 199-205, 2009.

[76] A. Kawa and S. Arakawa, "The effect of attenuated vaccinia virus AS strain on multiple myeloma: a case report," Japanese Journal of Experimental Medicine, vol. 57, no. 1, pp. 79-81, 1987. 

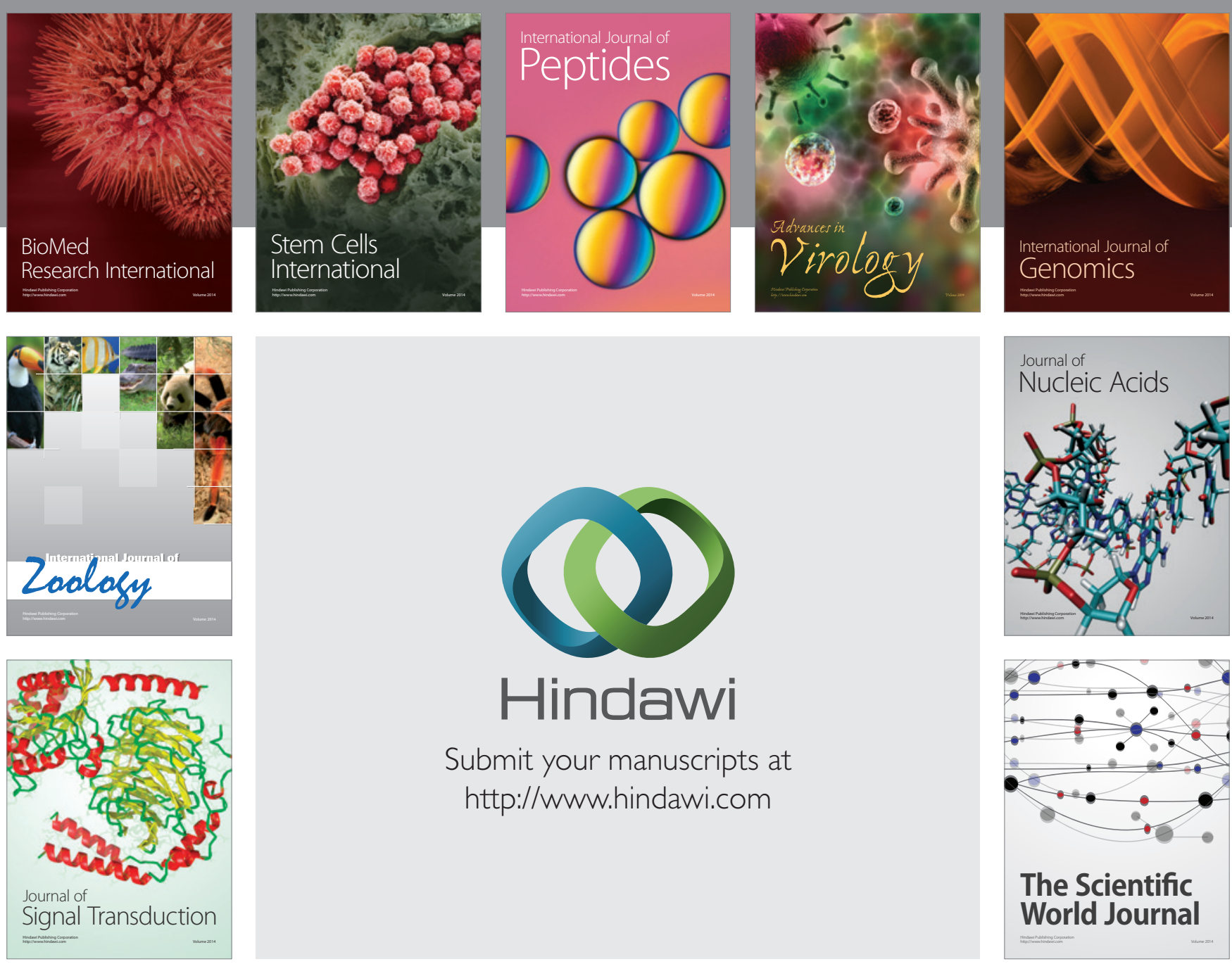

Submit your manuscripts at

http://www.hindawi.com
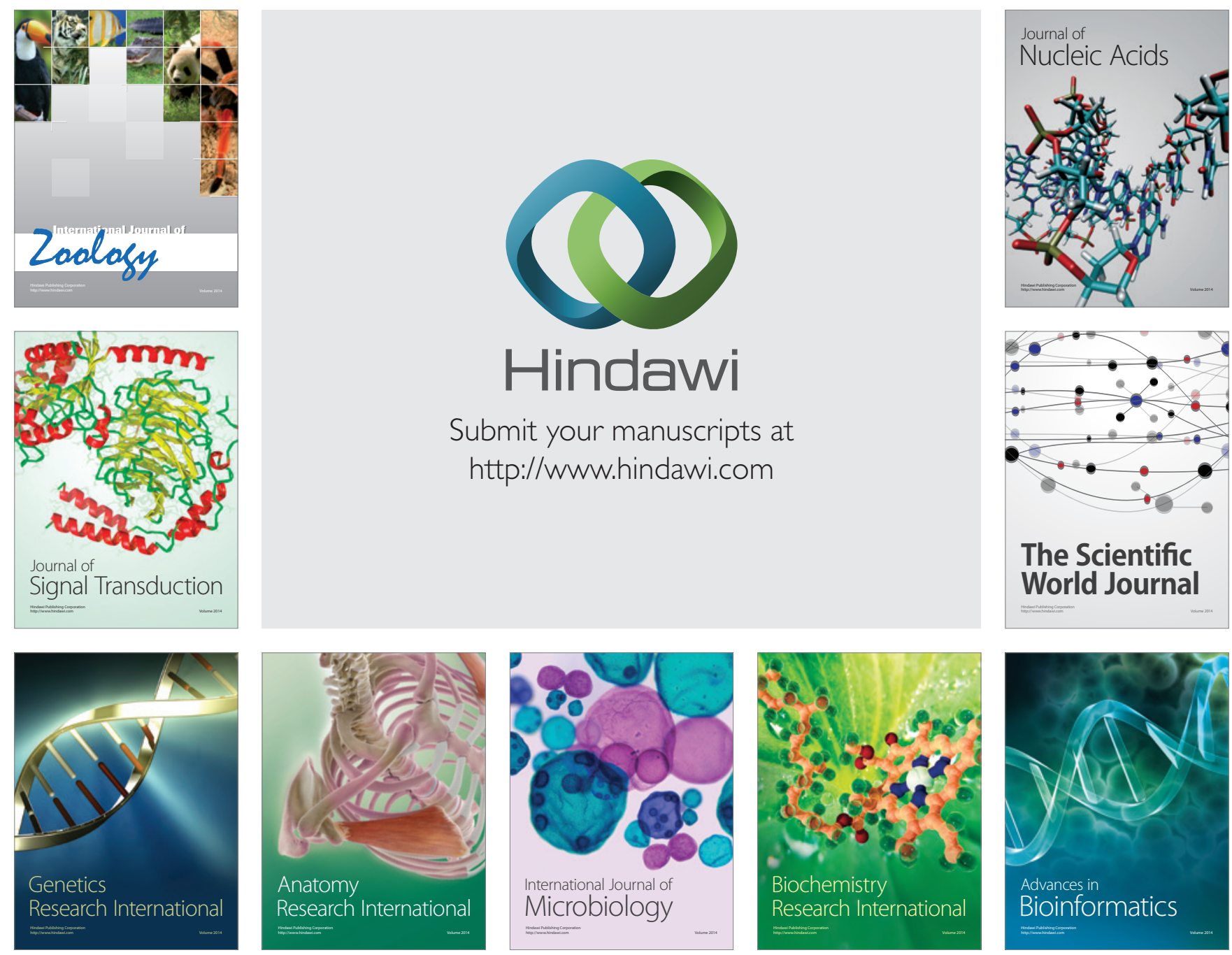

The Scientific World Journal
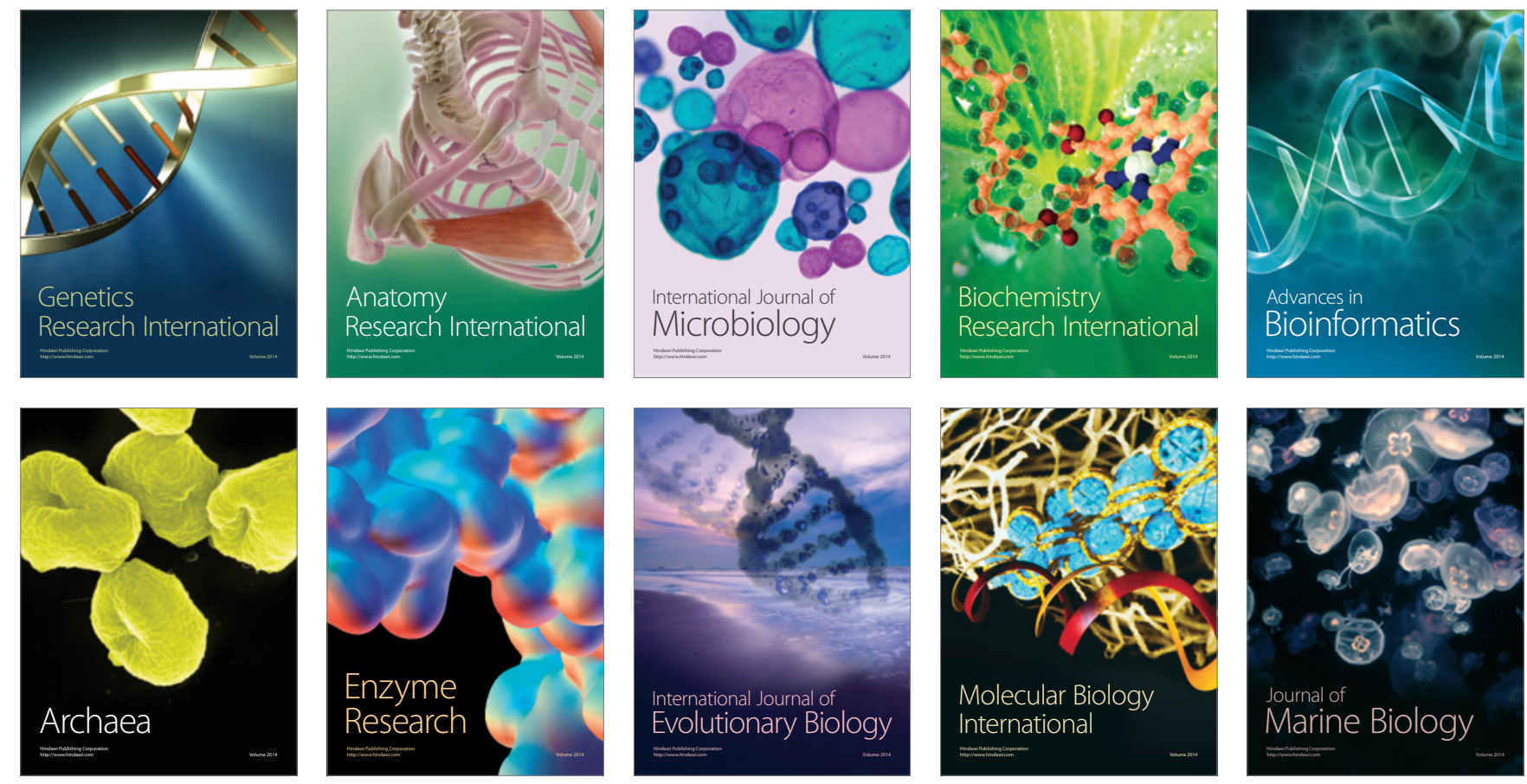